\title{
Extraretinal Control of Saccadic Suppression
}

\author{
Mark R. Diamond, ${ }^{1}$ John Ross, ${ }^{1}$ and M. C. Morrone ${ }^{2}$ \\ ${ }^{1}$ Department of Psychology, The University of WA, Nedlands Western Australia 6907, Australia, and 2/stituto di \\ Neurofisiologia del Consiglio Nazionale delle Ricerche, 56010 Pisa, Italy
}

We measured the time course of saccadic suppression and tested whether suppression results entirely from retinal image motion or has an extraretinal source. We measured contrast thresholds for low-frequency gratings modulated either in luminance, at $17 \mathrm{~cd} / \mathrm{m}^{2}$ and $0.17 \mathrm{~cd} / \mathrm{m}^{2}$, or color at $17 \mathrm{~cd} / \mathrm{m}^{2}$. Gratings were flashed on a uniform background before, during, luminance modulated gratings, saccades simulated by mirror motion.

A 10-fold decrease in contrast sensitivity was found for luminance-modulated gratings with saccades, but little suppression was found with simulated saccades. Adding highcontrast noise to the display increased the magnitude and the duration of the suppression during simulated saccades but had little effect on suppression produced by real saccades.

Suppression anticipates saccades by $50 \mathrm{msec}$, is maximal at the moment of saccadic onset, and outlasts saccades by $\sim 50$ or after voluntary $12^{\circ}$ saccades and, additionally in the case of

msec. At lower luminance, suppression is reduced, and its course is shallower than at higher luminance.

Simulated saccades produce shallower suppression over a longer time course at both luminances. No suppression was found for chromatically modulated gratings.

Differences between real and simulated saccades in the magnitude and time course of sensitivity loss suggest that saccadic suppression has an extraretinal component.

We model the effects of saccades by adding a signal to the visual input, so as to saturate the nonlinear stage of visual processing and make detection of a test stimulus more difficult.

Key words: saccades; eye movements; saccadic suppression; magnocellular; corollary discharge; image motion; efference copy

As far as the site of suppression is concerned, existing evidence points to somewhere early in the visual pathway, with the lateral geniculate nucleus being the most likely candidate. The evidence derives from two sources: first, from intracellular and extracellular recordings from rabbit and cat lateral geniculate nuclei (Fischer et al., 1996; Zhu and Lo, 1996; Derrington and Felisberti, 1998); second, from psychophysical studies indicating that suppression takes place before any interaction between successive stimuli, both for signals that mask one another (Burr et al., 1994) and those that interact to produce a percept of motion (Burr et al., 1999). In the study by Burr et al. (1994), for instance, the effects of masks presented during saccades were found to be attenuated when compared with those of masks presented after saccades, suggesting that the saccade had acted to suppress the mask before its interaction with a test stimulus.

Our main purpose in the experiments reported here is to disentangle the effects of saccadic eye movements from those of image motion alone. In doing so, we extend the work of Burr et al. (1994), by including both an examination of visual sensitivity from well before to well after the time saccades are made and an experimental condition (similar to that used in Bridgeman, 1983) in which saccade-like image motion is produced in the absence of saccades.

We also present a model of saccadic suppression that throws light on the mechanism through which it is achieved and on the site at which suppression acts.

Received Dec. 13, 1999; revised Feb. 18, 2000; accepted Feb. 18, 2000

This work was supported by Australian Research Council Grant ARC A79532415, European Community Grant EC BIOCT96-1461, and Human Frontier Science Program Grant RG0149/1999-B.

Correspondence should be addressed to Mark R. Diamond at the above address. E-mail: markd@psy.uwa.edu.au.

Copyright (C) 2000 Society for Neuroscience $0270-6474 / 00 / 203449-07 \$ 15.00 / 0$

\section{MATERIALS AND METHODS}

Stimuli. Stimuli were generated by framestore (Cambridge Research Systems VSG4) under the control of a $100 \mathrm{MHz}$ i486 personal computer (PC) and were displayed on the face of a Hitachi color monitor (HM- 
4821D) with luminance linearization at 120 frames/sec and 600 lines/ frame. The visible area of the screen was $35 \times 26 \mathrm{~cm}$, subtending $38.6 \times$ $29.1^{\circ}$ at a viewing distance of $50 \mathrm{~cm}$. The screen was surrounded by a $90 \times 80 \mathrm{~cm}$ card lit to match the screen in luminance and chromaticity.

Unless otherwise stated, the stimulus in all experiments was a horizontally oriented sinusoidal grating of 0.04 cycles $/{ }^{\circ}$, symmetric around the horizontal midline of the monitor, and displayed within a vertically oriented Gaussian envelope having a space constant of $4.8^{\circ}$. Stimuli were usually displayed for a single frame.

Saccades were made parallel to the orientation of the grating stimulus so that no motion signal would be produced by any motion of the grating image on the retina.

In some conditions the screen was covered with patterned noise through which the stimulus had to be detected. To construct the pattern, the screen was divided into blocks 4 pixels wide $\times 4$ raster lines deep. Each block was assigned, at random, either maximum or minimum luminance with equal probability. The pattern itself appeared only on even-numbered raster lines leaving the odd-numbered lines free to display the stimulus to be detected.

The stimulus was modulated either in luminance (red + green) or in chromaticity (red - green) by simultaneously varying the output of the red and green guns of the monitor using the method described by Burr and Morrone (1993). Equiluminance for each observer was determined by flicker photometry, with the amplitude ratio of the red and green guns being adjusted to produce minimal perceived flicker when the stimulus was modulated at $15 \mathrm{~Hz}$. The equiluminant point was confirmed by determining the red-green amplitude ratio that resulted in the highest nonsaccadic thresholds for detection of the chromatically modulated stimulus.

Thresholds were measured using either detection or identification tasks. In detection tasks, the observer responded with "yes" or "no" to indicate whether the stimulus was or was not seen. In identification tasks, the stimulus was shown either with normal contrast polarity or in reversed polarity and the observer reported polarity. In detection tasks, some zero-contrast catch trials were included. Across all observers and conditions where detection tasks were used, the false alarm rate was $<1$ in 200 trials-low enough to eliminate the possibility of criterion shifts.

Stimuli were presented within an interval from $200 \mathrm{msec}$ before to 200 msec after the onset of saccades, their contrast varying according to a ZEST procedure (King-Smith et al., 1994), which independently estimated the most informative contrast at which to present the next stimulus for each intended 25 or $50 \mathrm{msec}$ epoch within the $400 \mathrm{msec}$ period of interest. Saccadic latencies were monitored to determine the optimal time, in relation to the appearance of the saccadic target, at which to present the stimulus on each trial.

The final estimate of the contrast threshold for each epoch was made by fitting a Weibull (1951) function to observers' response data. An indication of the error associated with each threshold estimate was obtained from a sample of 20 such thresholds by calculating the "leaveone-out" error estimator (Weiss and Kulikowski, 1991). The maximum root-mean-squared error was 0.037 log-units-smaller than the size of symbols used in the figures.

Eye movements. Voluntary saccadic eye movements were monitored with an infrared scleral limbus tracker (HVS SP150) that sampled eye position at $10 \mathrm{kHz}$ and averaged the record continuously over each 1 msec period. The PC in turn sampled the output of the limbus tracker at $1 \mathrm{kHz}$ and stored the result in digital form. Immediately after each trial, the time of onset and completion of the saccade, relative to the time of presentation of the stimulus, was determined by the PC using a method adapted from Koski et al. (1995).

The mirror. In all experiments except the first, observers viewed a reflection of the video monitor screen in a thin plastic mirror $30 \times 20 \mathrm{~cm}$. In saccade conditions, the mirror was kept still, and the observer made a saccade. In simulation conditions, the observer maintained a fixed direction of gaze, and the computer activated a solenoid to rotate the mirror around its central vertical axis (Fig. 1).

The motion of the mirror was monitored in a manner comparable to that used in monitoring eye movements, by tracking a small imitation eye spindle of the mirror with the infrared scleral limbus tracker. The time course of the artificial saccade produced by rotating the mirror compares favorably to that of the saccading human eye (Fig. 2). In both cases the image motion lasts for $\sim 60 \mathrm{msec}$, although the deceleration of the mirror is not quite as smooth as that of the eye.

Procedure. Observations were made in a dimly lit room with the observer seated and with his or her chin on a chin rest.

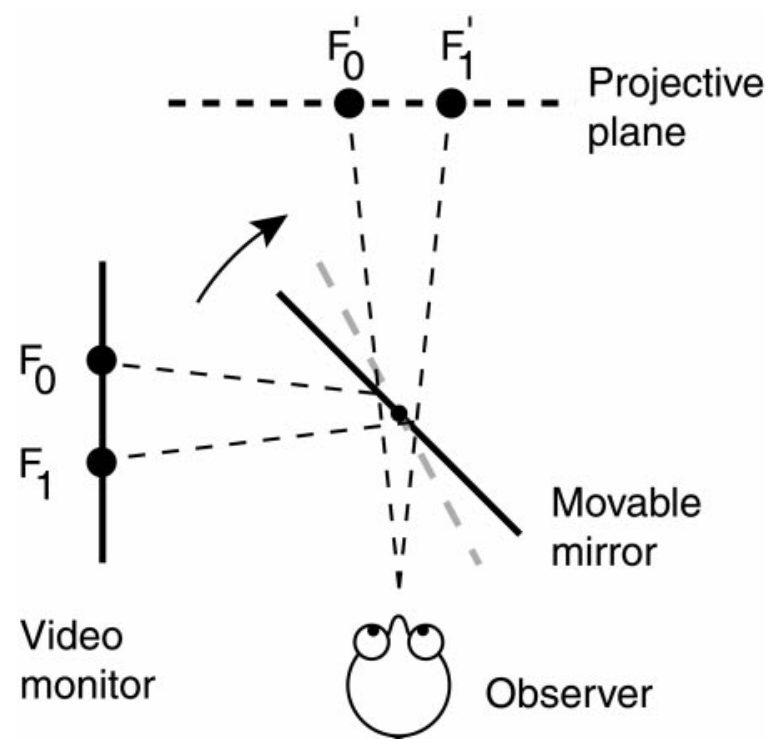

Figure 1. Viewing arrangement. In all but the first experiment (see Materials and Methods) observers viewed an image of the screen at an effective distance of $50 \mathrm{~cm}$. In conditions requiring simulated saccades, the mirror was rotated around its center to the position shown in gray. $F_{0}$ represents the observer's initial fixation; $F_{1}$ represents the saccadic target. The projective images of these points are shown as $F_{0}{ }^{\prime}$ and $\mathrm{F}_{1}{ }^{\prime}$.

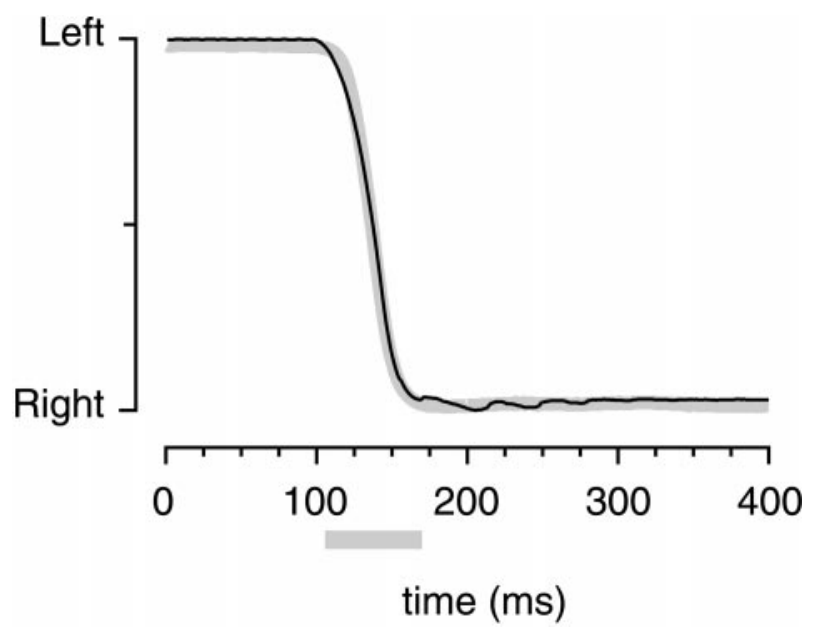

Figure 2. Traces, obtained from a scleral-limbus tracker, of the motion of a saccading eye (thick gray line) and an artificial eye mounted on a rotating mirror (black line). The traces overlap for most of their course but show that the deceleration of the mirror is not quite as smooth as that of the eye. The horizontal gray bar indicates the duration of image motion.

Each trial began with a $500 \mathrm{msec}$ warning tone followed by an interval randomly set between 800 and $1200 \mathrm{msec}$ during which time the observer fixated a $0.5^{\circ}$ spot, usually $6^{\circ}$ to the left of center of the screen. This fixation spot remained on throughout the trial. At some predetermined time, a target spot appeared on the screen $12^{\circ}$ to the right of the fixation point and then remained throughout the trial. In saccade conditions, the observer was required to make a saccade to the newly presented target, whereas in mirror movement conditions, the observer was required to maintain a steady gaze in the direction of the original fixation point while the mirror rotated. Although we did not monitor eye movements during trials requiring mirror motion, we had previously established that observers maintained a steady gaze under these conditions and that they did not track the moving image with their eyes.

As noted previously, stimuli were generally flashed on the screen for one $(8 \mathrm{msec})$ frame.

Observers. The authors, who at the time of the experiments ranged in 


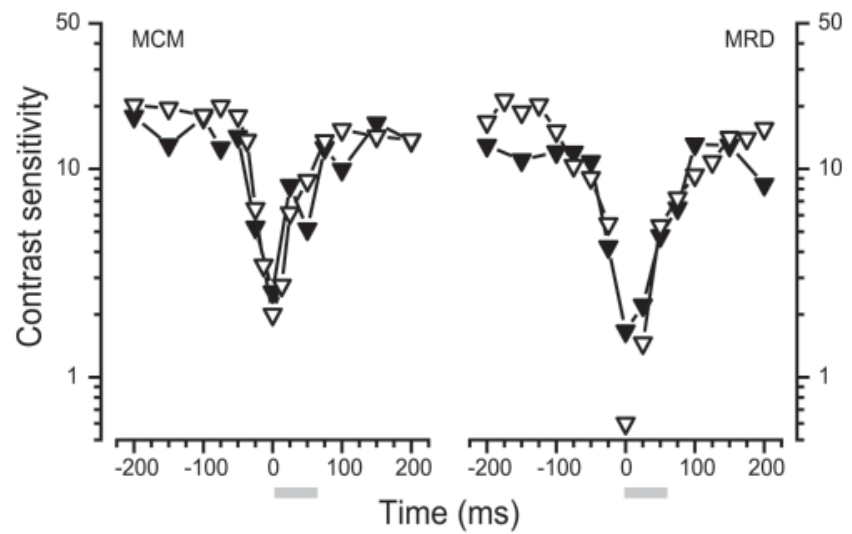

Figure 3. Contrast sensitivity under conditions in which the video screen was viewed directly (open triangles) or in a mirror (solid triangles). The horizontal gray bars indicate the duration of image motion; sensitivity values $<1$ indicate indeterminate thresholds. Error estimates calculated using leave-one-out resampling (Weiss and Kulikowski, 1991) are smaller than symbol size.

age from 40 to 66 years, served as observers. All have normal or corrected to normal vision.

\section{RESULTS}

\section{Saccades: direct versus mirrored viewing}

In the first experiment observers viewed a fixation spot $6^{\circ}$ to the left of the apparent screen center and made a saccade to an identical target spot $6^{\circ}$ to the right of center, as soon as possible after the spot appeared. In one condition observers viewed the screen directly from in front at a distance of $50 \mathrm{~cm}$. In the other condition they viewed its mirror image at an effective distance of $50 \mathrm{~cm}$. The luminance-modulated stimulus appeared on the screen-otherwise uniform except for the fixation and target spots-for one frame at a time, $t$, that was unpredictable by the observer. Presentation time was within the range of -200 to 200 msec from the start of the saccade $(t=0)$.

The time of presentation of the saccadic target was determined by both the observer's saccadic latencies and the desired time of presentation of the stimulus in relation to the onset of the observer's saccade.

Figure 3 shows the results of the first experiment. Both observers show very similar patterns of sensitivity for the two conditions, direct and mirrored observation. In both cases saccadic suppression is manifest $>50 \mathrm{msec}$ before saccades are made and outlasts them by at least $50 \mathrm{msec}$. The suppression is maximal precisely at the moment saccades begin. Sensitivity recovers while saccades are in progress and continues to do so after they have been completed.

\section{Saccades versus mirror motion Uniform screen}

In the second experiment observers always observed the screen in a mirror. In one condition they made a saccade as in Experiment 1 ; in the other they maintained fixation while the mirror moved, shifting the image of the screen. The mirror was moved to simulate closely the image motion caused by saccades (see Materials and Methods).

The two observers (MRD and MCM) showed very little loss of sensitivity when the mirror moved to shift the image of the screen while they were maintaining fixation (Fig. 4).

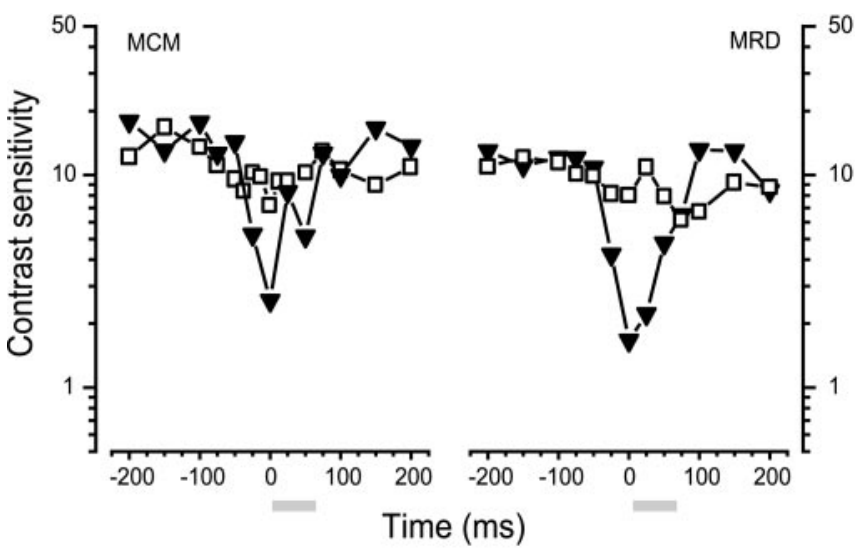

Figure 4. Contrast sensitivity during saccades (solid triangles) and during image motion caused by mirror rotation (open squares) in which the task was to identify the brightness polarity of the midline of a flashed grating. Contrast sensitivity during saccades drops by $\sim 1 \log$ unit and reaches a minimum at the time of onset of the saccade $(t=0)$. Changes in contrast sensitivity caused by the movement of the image alone are comparatively small. The horizontal gray bars indicate the duration of image motion.

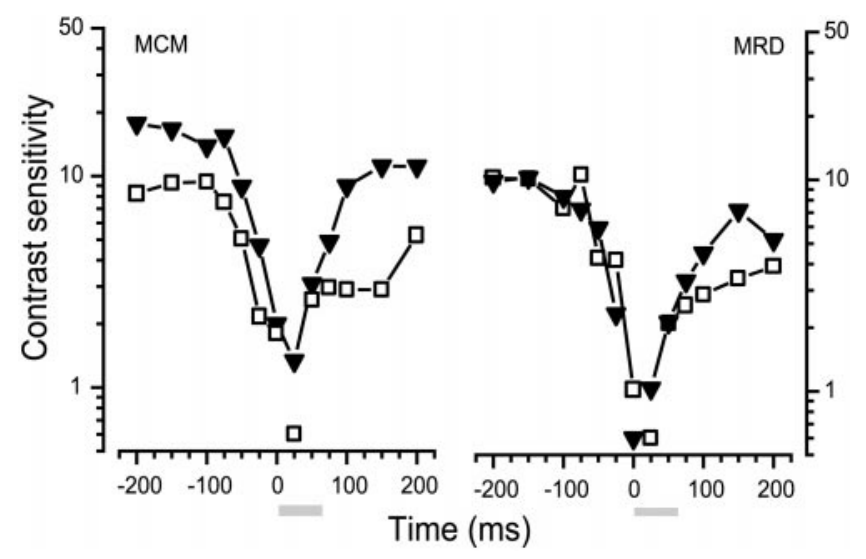

Figure 5. Contrast sensitivity during saccades (solid triangles) and during image motion caused by mirror rotation (open squares) where the task was to detect a grating flashed against a highly patterned background. Contrast sensitivity changes during saccades are almost identical with those shown in Figure 4. Changes in contrast sensitivity caused by the movement of the image alone follow a more extended time course of recovery to baseline. From approximately $+75 \mathrm{msec}$, contrast sensitivity after saccades exceeds that after mirror motion. The horizontal gray bars indicate the duration of image motion; sensitivity values $<1$ indicate indeterminate thresholds.

\section{Patterned noise}

The third experiment was identical to the second with two exceptions. First, the previously uniform screen was covered with a dense, high-contrast pattern (see Materials and Methods); second, the task for both observers was to detect the grating rather than report contrast polarity. The purpose of the patterned noise was to increase the strength of signals because of image motion, both during saccades and during mirror motion while fixation was maintained.

The results (Fig. 5) show that the presence of the pattern reduces overall sensitivity and makes a large difference to sensitivity loss when the mirror moves, but makes very little difference to the effects of saccades. Sensitivity loss for mirror motion is considerably larger with the patterned screen than with a uniform screen (Fig. 4) and is comparable in magnitude to that for sac- 


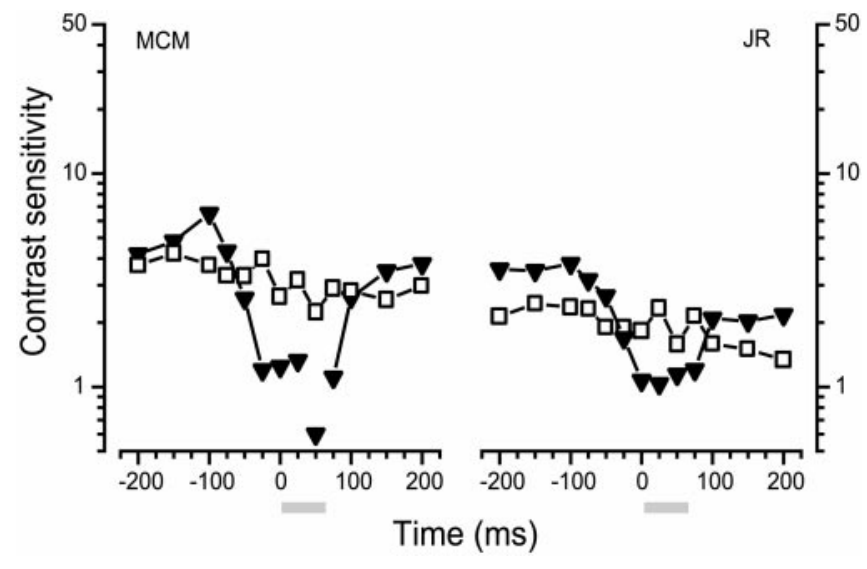

Figure 6. Contrast sensitivity at low luminance $\left(0.17 \mathrm{~cd} / \mathrm{m}^{2}\right)$ during saccades (solid triangles) and during image motion caused by mirror rotation (open squares). For MCM, the task was to identify the contrast polarity of a flashed grating, for JR it was to detect the grating. The saccade is associated with a loss in contrast sensitivity of $\sim 0.5 \log$ units, whereas there is virtually no loss in sensitivity associated with mirror motion. The horizontal gray bars indicate the duration of image motion; sensitivity values $<1$ indicate indeterminate thresholds.

cades. More remarkable is that the recovery of sensitivity is slower for mirror motion than for saccades, with both observers showing lower sensitivity after mirror motion than after saccades for times in the range of 100-200 msec. Because retinal image motion is identical when saccades are made and when the mirror moves, the lower sensitivity after mirror motion than after saccades indicates that preparation for saccades serves to counteract the aftereffects of image motion.

\section{Low luminance}

The fourth experiment was again identical to the second except that observers viewed the screen (in a mirror) through two log-unit neutral density filters that reduced screen luminance from 17 to $0.17 \mathrm{~cd} / \mathrm{m}^{2}$. As Figure 6 shows, there is a reduction in overall sensitivity for observer MCM of $\sim 0.5 \log$ units when compared with that found in conditions of high luminance (Fig. 4). The sensitivity loss accompanying saccades is also less at the low luminance level, although still substantial; here it is $\sim 0.6 \log$ units compared with 1 log unit in high-luminance conditions. This result is in keeping with that of Burr et al. (1982), who found that saccadic suppression was reduced at low luminance. Finally, the results show little sign of any effect of mirror motion on sensitivity.

\section{Equiluminance}

The final experiment examined the time course of sensitivity when saccades were made to stimuli modulated in color at equiluminance. A related experiment by Burr et al. (1994) showed no evidence of saccadic suppression from a point $20 \mathrm{msec}$ after saccadic onset, but left open the possibility there had simply been a rapid recovery in contrast sensitivity from time $t=0 \mathrm{msec}$. Also unexamined was the course of sensitivity in the period immediately preceding the saccade.

The results (Fig. 7) for MCM show a similar enhancement in sensitivity to that reported in Burr et al. (1994), although the point of peak sensitivity in the current experiment appears $\sim 25$ msec later than that reported 6 years ago. The results for MRD also show some enhancement in sensitivity, peaking at approximately $t=125 \mathrm{msec}$, although there is also a slight drop in

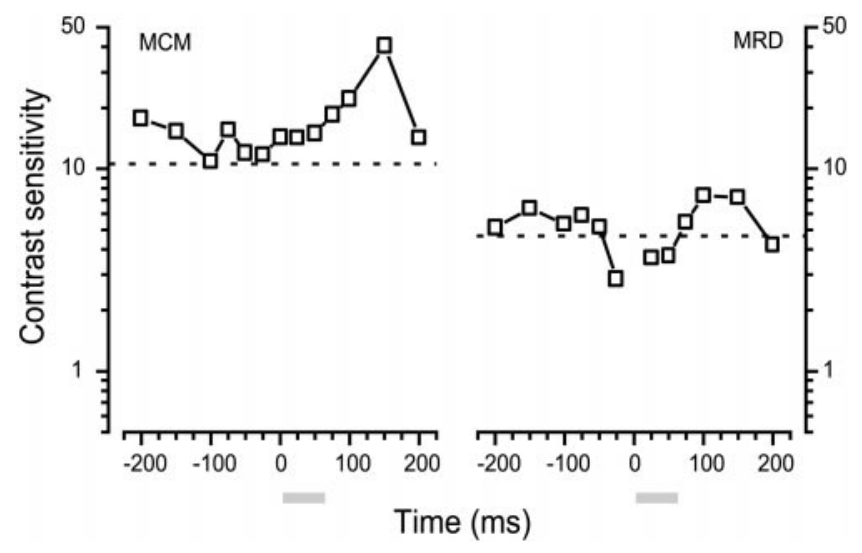

Figure 7. Contrast sensitivity during saccades for gratings modulated in color (red-green) at equiluminance. The dotted line represents contrast sensitivity in the absence of saccades or mirror motion. For both observers, there was some suggestion of presaccadic enhancement of sensitivity and no sign of the strong saccadic suppression found with stimuli modulated in luminance. The sharp peak in sensitivity for MCM at $150 \mathrm{msec}$ is very similar to that reported in Burr et al. (1994). The horizontal gray bars indicate the duration of image motion.

sensitivity just before saccadic onset. In addition, the results for both observers suggest the possibility of a previously undocumented presaccadic enhancement of contrast sensitivity to gratings modulated in color.

\section{Model}

Our model of saccadic suppression is shown in schematic form in Figure 8 . The flashed horizontal grating that served as our test stimulus is modeled as a delta function, $T(t)$, of variable contrast at the various perisaccadic times. The visual input produced by motion of the patterned screen background, whether as a result of mirror rotation or saccades, is modeled as a rectangular function, $N(t)$, set high from time 0 to time of $57 \mathrm{msec}$, reflecting the duration of mirror rotation and of saccades. The amplitude of the rectangular profile was a free parameter of the model, and, in the case of the uniform screen was zero.

The choice of a rectangular function is justified by an analysis of variance of the luminance profile of each point on the retina over time. When the mirror and the eye are both stationary, the luminance variation over a short time is zero, whereas when the mirror is moved or a saccade is made, the illumination of any point on the retina will change rapidly, around some mean value, as the myriad high and low luminance pixels sweep across it. The average variance over retinal position and over trials will be constant during the image motion.

In the first part of the model, the impulse response function of the visual system is represented as the difference of two $n$-stage filters (Swanson et al., 1987). Its form is given by:

$$
F(t)=A t^{n-1} e^{-\alpha t}-B\left(t-t_{0}\right)^{n-1} e^{-\gamma\left(t-t_{0}\right)},
$$

where $n$ is equal to $5, t_{0}$ is the time delay of the second filter, equal to $76 \mathrm{msec}$, and $\alpha$ and $\gamma$ are the time constants of the filters, equal to 58 and $75 \mathrm{~Hz}$, respectively. The parameters $A$ and $B$ govern the gain of each filter, and in our model have a ratio of 5.25:1. The final shape of the impulse response is similar to that derived by Swanson et al. (1987) (see also Burr and Morrone, 1996) at 9 trolands, although we have slightly adjusted parameter values to produce a simultaneous fit of the model to data for observers MCM and MRD. 


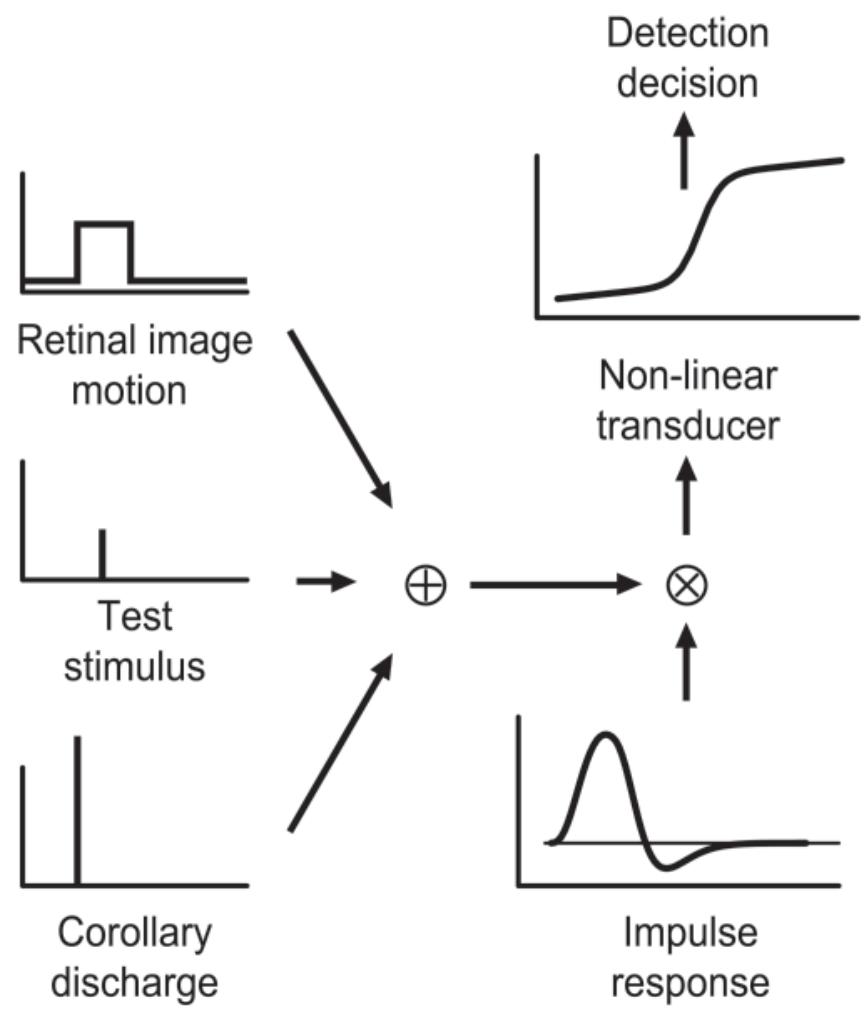

Figure 8. A model of saccadic suppression. The contrast change resulting from retinal image motion is added to that resulting from the presentation of the test stimulus. When saccades are made, a corollary discharge is also added to the early input. The input is convolved with the impulse response function of the visual system, and the output of the convolution is in turn passed through a nonlinear transducer. The output of the transducer serves as the basis for a decision about the presence or absence of the test stimulus (see "Model").

To obtain the linear response of the first stage of the model in conditions when no saccade is made, the test and the patterned noise are each convolved with the impulse response of the visual system, giving:

$$
\begin{aligned}
& R_{T}(t)=F(t) \otimes T(t), \\
& R_{N}(t)=F(t) \otimes N(t),
\end{aligned}
$$

and, as a consequence our assumption of linearity,

$$
R_{N+T}(t)=R_{N}(t)+R_{T}(t) .
$$

The nonlinear responses, $O_{\mathrm{N}}(t)$ for the noise pattern alone and $O_{N+T}(t)$ for the test superimposed on the pattern, are then obtained by applying the standard Naka and Rushton (1966) transformation to the previously obtained linear outputs:

$$
\begin{aligned}
O_{N}(t) & =\frac{R_{N}^{2}}{R_{N}^{2}+C^{2}}, \\
O_{N+T}(t) & =\frac{R_{N+T}^{2}}{R_{N+T}^{2}+C^{2}} .
\end{aligned}
$$

A threshold is established if the difference between the response to the pattern alone and the response to the patternplus-test reaches a predetermined, constant value after taking into account probability summation (Pelli, 1987). This is equivalent to stating that:

$$
\left(\sum_{t}\left|O_{N+T}(t)-O_{N}(t)\right|^{\beta}\right)^{\frac{1}{\beta}}>k
$$

where $k$ is a constant that determines the threshold, and $\beta$ is the slope of the psychometric function that determines the steepness of the probability summation.

To simulate the difference between the effects of image motion in the presence of saccades and image motion without saccades, it is sufficient to consider a central signal (labeled "corollary discharge" in Fig. 8) included in the input to the early, linear portion of the visual system. A delta function, $S(t)$, that mimics the action of the corollary discharge is added to the rectangular function that mimics the effect of image motion, transforming Equation 3 to:

$$
R_{N+T+S}(t)=R_{N}(t)+R_{T}(t)+(F(t) \otimes S(t)) .
$$

If the corollary discharge is sufficiently brief, it will generate a transient suppression. The spike representing the corollary discharge effectively saturates the nonlinear transducer and reduces the sensitivity of the visual system to the test stimulus by reducing the differences between $O_{\mathrm{N}}(t)$ and $O_{N+T}(t)$ that are summed in Equation 6.

\section{Fit to the data}

The continuous curves in Figure 9 show the inverse of the minimum amplitude of the test required to satisfy Equation 6 under each of three conditions: saccades with a uniform background (Fig. 9a), saccades with a patterned background (Fig. 9b), and mirror motion with a patterned background (Fig. 9c). The same parameters, except for an overall multiplicative scaling, were used for both subjects, yielding a good fit with a maximum variation of $<4 \mathrm{~dB}$.

The curves in Figure 9, $a$ and $b$, show the results of the model when a signal of the kind described in the preceding subsection - a delta function at time 0 -is included both for the uniform screen condition and the patterned screen condition. The model reproduces the major characteristics of the time course of suppression, with the peak decrement in sensitivity occurring at $t=0$, followed by a gradual and prolonged recovery.

In the case of image motion without saccades (Fig. 9c), the model is again capable of accounting for the prevailing features of the time course of visual sensitivity: namely that sensitivity loss starts $\sim 75 \mathrm{msec}$ before any image motion takes place and endures until $\sim 100 \mathrm{msec}$ after the motion stops. At approximately +25 msec, the model predicts a physically unrealizable threshold (log contrast, $>5$ ); interestingly, for those times it was also impossible to determine a psychophysical threshold because the subjects' responses were at chance for the maximum contrast of the test.

Although not shown in Figure 9, the model also correctly predicts the absence of sensitivity loss that is found with mirror motion together with a uniform screen.

\section{DISCUSSION}

Our results indicate that saccades have an effect on contrast sensitivity that is remarkably consistent across changes in luminance and image structure (patterned vs uniform screen). In contrast, the effects of image motion in the absence of saccades depend strongly on characteristics of the visual image.

For saccades, the general picture is that sensitivity loss begins 

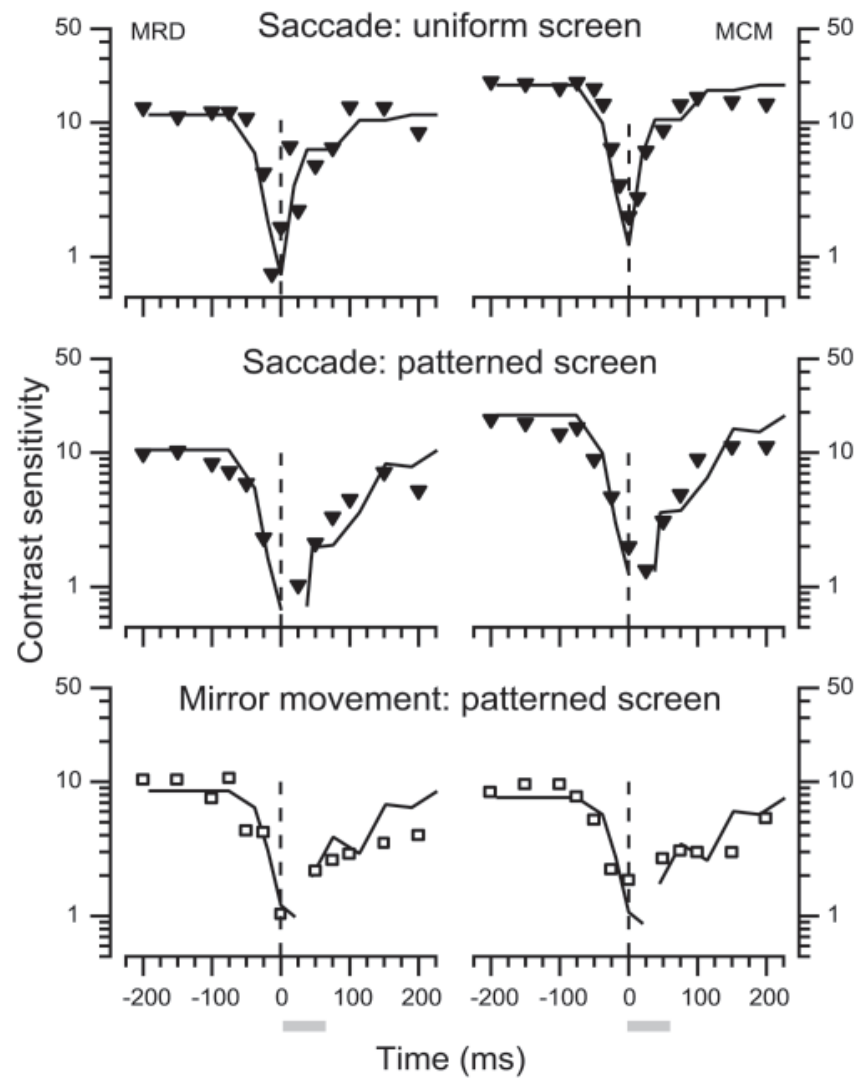

Figure 9. Fit of the model to the experimental data for saccades with a uniform screen $(a)$, saccades with a patterned screen $(b)$, and mirror motion with a patterned screen $(c)$. Solid triangles indicate data points for saccadic conditions, open squares indicate data points for mirror motion. The solid lines in all panels represent the predictions from the model described in Model.

$\sim 75$ msec before the onset of any retinal image motion, is maximal at the moment of motion onset, and outlasts the saccade by $\sim 50 \mathrm{msec}$.

Although non-saccadic image motion can produce a somewhat similar pattern of sensitivity loss, such a pattern only appears when luminance is high and the image is highly textured. Even then, there are differences between the effects of saccades and nonsaccadic image motion, most notably in the somewhat slower recovery of sensitivity in the no-saccade condition.

Our model places the site of action for saccadic suppression early in the visual pathway, before a nonlinear stage of signal transduction. It assumes the visual system responds to motion signals caused by eye movement and also to a signal of extraretinal origin. These provide a context within which a stimulus is to be detected. Detection is successful when the contrast of the stimulus is high enough to produce a level of activity that, when integrated over time, exceeds by a sufficient amount the activity that results when the stimulus is absent.

The inclusion of a corollary discharge as part of the input, taken together with a spreading of activity in time and a nonlinear stage (see model), has two effects. First, there is a predicted sensitivity loss for stimuli presented before saccades, consistent with our results. Second, predicted recovery is faster after saccades than after image motion in the absence of saccades. The interaction of retinal input and corollary discharge blunts the effects that motion alone would have had. The corollary discharge provides the visual system with some immunity to image motion, and perhaps to awareness of it.

\section{Suppression and compression}

The time course of saccadic suppression is very similar to that of spatial compression (Morrone et al., 1997; Ross et al., 1997) in which targets flashed around the time of saccades are seen as displaced in external space toward their targets. Like suppression, compression appears before saccades, is maximal at the time they start, reduces while they are in progress, and finally disappears only after they have ended. Apparent position is shifted when images are moved at saccadic speeds by a mirror, but there is no spatial compression (Morrone et al., 1997). The similarity of the time courses of the two effects of saccades, suppression and compression, suggests they are both driven by a common mechanism, presumably the spike of corollary discharge assumed in our model. This signal may not only reduce sensitivity within the magnocellular pathway, but may also trigger an alteration in the cortical mapping of external space. Although our model assumes a signal at time $t=0$, the work of Duhamel's group provides evidence that some lateral intraparietal area (LIP) neurons shift their receptive fields before saccadic onset (Duhamel et al., 1992; Colby et al., 1995). As our model shows, impulse response functions can spread effects in time, and these may account for apparent differences in the timing of the added signal in our model and that responsible for alterations in the response characteristics of LIP neurons. Ultimately, however, the resolution of any apparent discrepancy must await the determination of the source, time course, and pathway of the putative corollary discharge.

The task of the visual system is to preserve stability as gaze is shifted and retinal images of the world moved by saccades. Corollary discharge may coordinate two main functional changes serving this purpose: a change of gain that reduces sensitivity to image motion, providing some immunity to the effects it would otherwise have had; and a temporary expansion and displacement of receptive fields that allows a smooth shift of coordinates, at the price of transitory spatial compression. The price is a small one to pay because compression affects only transitory targets that flash into view close to the time saccades are made, not objects that remain in view (Cai et al., 1997).

\section{REFERENCES}

Beeler GW (1967) Visual threshold changes resulting from spontaneous saccadic eye movements. Vision Res 7:769-775.

Bridgeman B (1983) Mechanisms of space constancy. In: Spatially oriented behavior (Hein A, Jeannerod M, eds), pp 263-279. New York: Springer.

Burr DC, Morrone MC (1993) Impulse response functions for chromatic and achromatic stimuli. J Opt Soc Am A 10:1706-1713.

Burr DC, Morrone MC (1996) Temporal impulse response functions for luminance and color during saccades. Vision Res 36:2069-2078.

Burr DC, Holt J, Johnstone JR, Ross J (1982) Selective depression of motion selectivity during saccades. J Physiol (London) 333:1-15.

Burr DC, Morrone MC, Ross J (1994) Selective suppression of the magnocellular visual pathway during saccadic eye movements. Nature 371:511-513.

Burr DC, Morgan MJ, Morrone MC (1999) Saccadic suppression precedes visual motion analysis. Curr Biol 9:1207-1209.

Cai RH, Pouget A, Schlag-Rey M, Schlag J (1997) Perceived geometrical relationships affected by eye-movement signals. Nature 386:601-604.

Campbell FW, Wurtz RH (1978) Saccadic omission: why we do not see a greyout during a saccadic eye movement. Vision Res 18:1297-1303. 
Colby CL, Duhamel JR, Goldberg ME (1995) Oculocentric spatial representation in parietal cortex. Cereb Cortex 5:470-481.

Derrington AM, Felisberti F (1998) Peripheral shift reduces visual sensitivity in cat geniculate neurones. Vis Neurosci 15:875-880.

Duffy FH, Lombroso CT (1968) Electrophysiological evidence for visual suppression prior to the onset of a voluntary saccadic eye movement. Nature 218:1074-1075.

Duhamel J-R, Colby CL, Goldberg ME (1992) The updating of the representation of visual space in parietal cortex by intended eye movements. Science 255:90-92.

Fischer WH, Schmidt M, Stuphorn V, Hoffman KP (1996) Response properties of relay cells in the A-laminae of the cat's dorsal lateral geniculate nucleus after saccades. Exp Brain Res 110:435-445.

Grüsser O-J (1994) Early concepts on efference copy and reafference. Behav Brain Sci 17:262-265.

Holt EB (1903) Eye movements and central anaesthesia. Psychol Rev 4:3-45.

Ilg UJ, Hoffmann K-P (1993) Motion perception during saccades. Vision Res 33:211-220.

King-Smith PE, Grigsby SS, Vingrys AJ, Benes SC, Supowit A (1994) Efficient and unbiased modifications of the QUEST threshold method: theory, simulations, experimental evaluation and practical implementation. Vision Res 34:885-912.

Koski A, Juhola M, Meriste M (1995) Syntactic recognition of ECG signals by attributed finite automata. Pattern Recognition 28:1927-1940.

MacKay DM (1970) Elevation of visual threshold by displacement of visual images. Nature 225:90-92.

Matin E (1974) Saccadic suppression: a review and an analysis. Psychol Bull 81:899-917.

Morgan MJ (1994) Vision. When it pays not to see. Nature 371:473.

Morrone MC, Ross J, Burr DC (1997) Apparent position of visual targets during real and simulated saccadic eye movements. J Neurosci 17:7941-7953.
Naka KI, Rushton WA (1966) S-potentials from colour units in the retina of fish (Cyprinidae). J Physiol (Lond) 185:536-555.

Pelli DG (1987) On the relation between summation and facilitation. Vision Res 27:119-123.

Ross J, Burr DC, Morrone MC (1996) Suppression of the magnocellular pathways during saccades. Behav Brain Res 80:1-8.

Ross J, Morrone MC, Burr DC (1997) Compression of visual space before saccades. Nature 384:598-601.

Shiori S, Cavanagh P (1989) Saccadic suppression of low-level motion. Vision Res 29:915-928.

Sperling G (1990) Comparison of perception in the moving and stationary eye. In: Eye movements and their role in visual and cognitive processes. (Kowler E, ed), pp 307-351. Amsterdam: Elsevier.

Sperry RW (1950) Neural basis of the spontaneous optokinetic response produced by visual inversion. J Comp Physiol Psychol 43:482-489.

Swanson WH, Ueno T, Smith VC, Pokorny J (1987) Temporal modulation sensitivity and pulse-duration thresholds for chromatic and luminance perturbations. J Opt Soc Am A Opt Image Sci Vis 4:1992-2005.

Uchikawa K, Sato M (1995) Saccadic suppression to achromatic and chromatic responses measured by increment-threshold spectral sensitivity. J Opt Soc Am A 12:661-666.

Von Holst E, Mittelstaedt H (1954) Das Reafferenzprinzip. Naturwissenschaften 37:464-476.

Weibull W (1951) A statistical distribution function of wide applicability. J Appl Mech 18:292-297.

Weiss SM, Kulikowski CA (1991) Computer systems that learn: classification and prediction methods from statistics, neural nets, machine learning and expert systems. San Mateo, CA: Morgan Kaufmann.

Zhu JJ, Lo FS (1996) Time course of inhibition induced by a putative saccadic suppression circuit in the dorsal lateral geniculate nucleus of the rabbit. Brain Res Bull 41:281-291.

Zuber BL, Stark L, Lorber M (1966) Saccadic suppression of the pupillary light reflex. Exp Neurol 14:351-370. 